Revista de la Escuela de Ciencias de la Educación, año 14, nRo. 13, vol. 1, enero a Junio de 2018. Páginas 167-179. ISSN 1851-6297 (DESDE DICIEMBRE DE 2006 A DICIEMBRE DE 2017). ISSN 2362-3349 (EN LINEA). LA INTERACCIÓN EN LA CLASE DE LENGUA Y LITERATURA: ANÁLISIS DEL DIÁLOGO. DoRA RIESTRA.

\title{
LA INTERACCIÓN EN LA CLASE DE LENGUA Y LITERATURA: ANÁLISIS DEL DIÁLOGO
}

\author{
Por Dora Riestra* \\ Universidad Nacional de Río Negro, Argentina \\ dora.riestra@gmail.com
}

Recibido: 02/10/2107 Aceptado: 20/11/2017

\section{Resumen}

Desde el proyecto de investigación "Modelos didácticos disociados: ¿enseñar lengua o enseñar literatura?" (PI UNRN 40-B- 362) buscamos conocer cuáles son las consecuencias de esta separación curricular en el aula. Este tipo de investigaciones conjuga la relación enseñanza-aprendizaje como interacción que busca producir efectos a través de las consignas de trabajo. El análisis de los efectos se realiza en función de la formación de profesores y en función de la revisión de los objetos de enseñanza de la lengua primera.

Por otra parte, de los análisis realizados hasta el momento en el marco del Interaccionismo socio-discursivo, hemos detectado dificultades en la comunicación en el aula y, en consecuencia, estamos desarrollando un dispositivo de análisis del diálogo desde el enfoque de Jakubinskij (2015), con la finalidad de aportar al análisis del trabajo del profesor.

En este artículo se presentan algunos aspectos metodológicos que consideramos relevantes, sobre los que estamos trabajando.

* Es Profesora en Letras para la Enseñanza Media y Superior, Universidad Católica de Santa Fe (1969). Docteure en Sciences de l'Éducation, Université de Genève, Suiza (2004). Director: Jean-Paul Bronckart. Profesora adjunta regular en Usos y Formas de la Lengua Escrita, CRUB-Universidad Nacional del Comahue (1995-2015). Profesora titular regular de Lengua en Instituto de Formación Docente Continua de Bariloche (20042009). Profesora titular regular en Didáctica de la Lengua y la Literatura, Universidad Nacional de Río Negro (2009-2015).

Desde 2016 es Profesora consulta de la Universidad Nacional de Río Negro y realiza actividades de docencia en posgrado e investigación. También desarrolla la docencia en posgrado en diversas universidades nacionales y de Brasil. Investigadora Categoría I en Didáctica de la Lengua y la Literatura. 
Revista de la Escuela de Ciencias de la Educación, año 14, nRo. 13, vol. 1, enero a junio de 2018. Páginas 167-179. ISSN 1851-6297 (DESDE DICIEMBRE DE 2006 A DICIEMBRE DE 2017). ISSN 2362-3349 (EN LiNEA). LA INTERACCIÓN EN LA CLASE DE LENGUA Y LITERATURA: ANÁLISIS DEL DIÁLOGO. DORA RIESTRA.

Palabras clave: Enseñanza de la lengua - Interacción - Comunicación en clase - Análisis del diálogo - Trabajo docente.

\section{Abstract}

From the research project "Dissociated didactic models: teaching language or teaching literature?" (PI UNRN 40-B- 362) we seek to know the consequences of this curricular separation in the classroom. This type of research combines the teaching-learning relationship as an interaction that seeks to produce effects through the instructions of work. The analysis of the effects is done in function of the training of teachers and in function of the revision of the objects of teaching the first language.

On the other hand, from the analyzes carried out so far in the context of socio-discursive interactionism, we have detected difficulties in communication in the classroom and, consequently, we are developing a device for analyzing dialogue from Jakubinskij (2015), with the purpose of contributing to the analysis of the teacher's work.

This article presents some methodological aspects that we consider relevant, on which we are working.

Keywords: Teaching language - Interaction - communication in the classroom - The analysis of dialogue - The teacher's work.

\section{Introducción}

En las investigaciones que abordan la enseñanza y/o el aprendizaje en el aula, las posiciones epistemológicas han enfocado, mayoritariamente, las significaciones como producto del pensamiento, es decir, las significaciones serían individuales y cognitivas para transformarse después en semióticas y socio-culturales, a partir de un reformateo producido por los sistemas semióticos elaborados socialmente. Esta posición que, según Bronckart $(2002 ; 2004)$ comparten Piaget, Peirce y Chomsky, sería retomada o reformulada hoy por el cognitivismo en el marco de las llamadas neurociencias.

En nuestro caso, partimos de una posición epistemológica opuesta, que se basa en el lenguaje humano y sus empirias como objeto de estudio, en la tradición de Humboldt, Jakubinskij, Vygotski y Voloshinov.

La posición epistemológica acerca del diálogo de Lev Jakubinskij, publicada en 1923, se basó en algunos principios respecto del habla dialogal que, posteriormente, tendrían influencia en otros rusos, como Voloshinov y Vygotski. En primer lugar, el principio de la formación e interiorización del lenguaje externo comprende el concepto de actividad de lenguaje:

Como al inicio, durante el período de aprendizaje, cada actividad de lenguaje (en términos precisos sería "lenguajera") es una actividad compleja y no habitual. La actividad de lenguaje automática pertenece al tipo de acti- 
Revista de la Escuela de Ciencias de la Educación, año 14, nRo. 13, vol. 1, enero a junio de 2018. Páginas 167-179. ISSN 1851-6297 (DESDE DICIEMBRE DE 2006 A DICIEMBRE DE 2017). ISSN 2362-3349 (EN LINEA). LA INTERACCIÓN EN LA CLASE DE LENGUA Y LITERATURA: ANÁLISIS DEL DIÁLOGO. DORA RIESTRA.

vidades automáticas secundarias que se forman a partir de las actividades conscientes por medio de repetición, ejercicio y hábito (Jakubinskij, 2015, p.110).

Desde esta perspectiva, el lenguaje humano es universal, pero las lenguas son aleatorias y cambian permanentemente por el efecto del tiempo y de las masas hablantes (Saussure, 2004), por lo tanto, la interacción humana se produciría a través de la palabra como proceso de semiosis, necesario para entenderse y actuar (Bronckart, 2004).

Fue Lev Vygotski (2009) quien, a partir del concepto de signo lingüístico saussureano elaboró su tesis acerca de la significación y la palabra (slovo en ruso) pronunciada por otro, como la herramienta externa que penetra en el individuo y permite la formación de la función psíquica superior, desarrollándose, de este modo, a través de la actividad de hablar, la capacidad de pensar (autorreflexividad humana) (Riestra 2014).

De acuerdo con este enfoque interaccionista socio-discursivo, las interacciones en clase de los distintos niveles de enseñanza constituyen el objeto de estudio de nuestras investigaciones empíricas.

En la actualidad, desde el proyecto de investigación "Modelos didácticos disociados: ¿enseñar lengua o enseñar literatura?" (PI UNRN 40-B- 362) buscamos conocer cuáles son las consecuencias de esta separación curricular en el aula. Este tipo de investigaciones conjuga la relación enseñanza-aprendizaje como interacción que busca producir efectos a través de las consignas de trabajo. El análisis de los efectos se realiza en función de la formación de profesores y en función de la revisión de los objetos de enseñanza de la lengua primera. Analizamos 30 clases de Lengua y literatura de los dos niveles obligatorios de enseñanza, 15 de primario y 15 de secundario.

Después del análisis de las consignas y el análisis del contenido de las clases, realizamos en el equipo de investigación, con profesoras de nivel secundario, dos secuencias didácticas con la finalidad de integrar la enseñanza de la lengua y la literatura.

El análisis de la aplicación de las secuencias muestra cómo, en cada clase, el objeto de enseñanza es presentado a los alumnos, a través de las consignas de las docentes y cómo los alumnos realizan las tareas propuestas en las consignas.

Por otra parte, ante las dificultades de comprensión de las tareas propuestas a los alumnos, realizamos un análisis del diálogo en la interacción de las clases para entender cómo se produce la comunicación entre profesores y alumnos en clase.

\section{Sobre el concepto de diálogo}

El concepto de diálogo que adoptamos es el de Jakubinskij y, cabe señalar que fue la lingüista rusa Irina Ivanova quien tradujo del ruso al francés en 
Revista de la Escuela de Ciencias de la Educación, año 14, nRo. 13, vol. 1, enero a junio de 2018. Páginas 167-179. ISSN 1851-6297 (DESDE DICIEMBRE DE 2006 A DICIEMBRE DE 2017). ISSN 2362-3349 (EN LiNEA). LA INTERACCIÓN EN LA CLASE DE LENGUA Y LITERATURA: ANÁLISIS DEL DIÁLOGO. DORA RIESTRA.

2012 el artículo de este autor titulado "Sobre el habla dialogal", texto que llegó a nosotros en portugués, en edición brasileña de 2015, revisada por Sériot e Ivanova.

El carácter de lo social como determinante está presente en los autores rusos de los años 20-30 y, si bien Jakubinskij parte de un análisis funcional, no es la "funcionalidad" de Jakobson, ligada al propósito o finalidad del lenguaje, sino la "diversidad funcional" humboldtiana, que distingue "diversidad de formas". La diversidad funcional fue observada en diálogos reales, es decir, prestó atención a la fonación antes de que la sociolingüística surgiera como enfoque disciplinar. La tradición rusa heredada del siglo XIX con enfoque lingüístico formal y psicológico, a partir de la fonética y de la actividad de lenguaje enfoca el diálogo con un nivel de análisis que aún hoy mantiene vigencia y permite esbozar perspectivas nuevas en la investigación.

A Jakubinskij le interesó el habla dialogal como "fenómeno y como forma no mediatizada", que comparó con otras formas mediatizadas como la escritura y el monólogo. Lo que observó fue la percepción auditiva y visual, por lo que abordó el diálogo como proceso de hablar. Delimitó con precisión descriptiva y funcional el automatismo verbal en la réplica, el interlocutor, el papel de la retención en tanto percepción y comprensión, aspectos que anticiparon el análisis conversacional de Sacks (1974) y el cognitivismo actual (Paas, Renkl y Sweller, 2004).

La diversidad funcional para Jakubinskij consiste en analizar diálogos reales en contraposición con monólogos (orales o escritos); el proceso de hablar es observado y definido como automatismo verbal (reacción natural espontánea de la réplica), por lo que el diálogo como fenómeno cultural está cercano a lo biológico ("psico-biológico", dirá él), es un "fenómeno de la naturaleza". Se posiciona de este modo ante el fenómeno del diálogo:

En nuestro caso, partiendo de la distinción de las formas de habla, establecemos un puente entre los factores extralingüísticos y los fenómenos verbales y tenemos, así, la posibilidad de hablar, por ejemplo, de la distinción de los medios de información en esa o aquella variante o de oponer directamente monólogo y diálogo como fenómenos verbales. (Jakubinskij, 2015, p.61. Traducción nuestra).

Jakubinskij describe: a) lo inacabado del diálogo (cada réplica supone una contra -réplica) y b) las interrupciones como necesarias en la interacción, ambas características señaladas entre otras, a partir de la observación empírica: "no es necesario saber interrumpir a alguien que habla porque eso es muy natural" (Jakubinskij, 2015, p.77).

Ivanova (2003/2010) menciona que los conceptos de percepción y apercepción que el autor utilizó para su análisis del diálogo tienen como referencia los desarrollos teóricos de William James, que le sirvieron para explicar los 
Revista de la Escuela de Ciencias de la Educación, año 14, nRo. 13, vol. 1, enero a junio de 2018. Páginas 167-179. ISSN 1851-6297 (DESDE DICIEMBRE DE 2006 A DICIEMBRE DE 2017). ISSN 2362-3349 (EN LINEA). LA INTERACCIÓN EN LA CLASE DE LENGUA Y LITERATURA: ANÁLISIS DEL DIÁLOGO. DoRA RIESTRA.

aspectos rítmicos del diálogo a los que llamó "clisés o estereotipos", que reducen considerablemente la cantidad de palabras:

al hablar del fenómeno de la apercepción, Jakubinskij llegó a la formulación de otro principio de la teoría del diálogo, a saber, la cuestión del papel de esos clisés (sablony) en la interacción. Jakubinskij liga los clisés (el estereotipo) de la situación cotidiana a los enunciados clisés. (Jakubinskij, 2015, p.50).

La noción de estereotipo o clisé es interesante y muy actual para analizar la interacción como proceso de comunicación en un contexto determinado, ya que se trata de la forma vivida, en el sentido de la "forma percibida", que incluye la entonación, la forma compartida, se define a través del carácter social de las formas.

El aspecto formal del análisis de los lingüistas y psicólogos rusos es complejo y abarcativo, ya que comprende tanto la actividad del lenguaje, como el pensamiento y la conciencia, los géneros, la dialogicidad y lo predicativo del habla interior, desde una perspectiva que no es la formal abstracta (Bertau, 2008).

\section{Las categorías de análisis}

A partir del concepto del habla dialogal automática de Jakubinskij elaboramos unas categorías para analizar las interacciones de las clases. Como puede verse en la Tabla 1, el concepto de masa aperceptiva de Jakubinskij como predisposición, sentido previo, está relacionado con las suposiciones y los prejuicios, tanto de los alumnos como de los profesores.

La utilidad de estas categorías para el análisis consiste en poder revisar lo automatizado del diálogo en nuestros propios enunciados como profesores. A la vez, se trata de poder visualizar las frases hechas y, en este sentido, los formatos automatizados que son los tipos discursivos (Bronckart, 1997; 2007), como construcciones cristalizadas de la acción de lenguaje que tenemos en común con los alumnos. Se trata del contar y el exponer, autónomo e implicado, que hemos internalizado desde las primeras interacciones verbales y tienen que ver con el uso de pronombres, deícticos y verbos conjugados.

Desde la investigación, proponemos una herramienta para analizar las diferentes lenguas funcionales (Coseriu, 1992) o sociolectos (Jakubinskij, 2015) de los profesores y los alumnos, en los que podemos distinguir los llamados estereotipos por Jakubinskij, que son las frases hechas del diálogo a las que normalmente no podemos prestar atención porque funcionan casi como gestos en las interrupciones o superposiciones de enunciados (característica específica del habla dialogal). En definitiva, el análisis consiste en una reflexión sobre lo automatizado del habla de la clase que nos permite analizar el trabajo docente desde nuestra perspectiva profesional. La finalidad de este tipo de análisis es la de poder tomar conciencia de nuestras formas de decir y las 
Revista de la Escuela de Ciencias de la Educación, año 14, nRo. 13, vol. 1, enero a junio de 2018. Páginas 167-179. ISSN 1851-6297 (DESDE DICIEMBRE DE 2006 A DICIEMBRE DE 2017). ISSN 2362-3349 (EN LiNEA). LA INTERACCIÓN EN LA CLASE DE LENGUA Y LITERATURA: ANÁLISIS DEL DIÁLOgo. DORA RIESTRA.

formas de decir de nuestros alumnos, las lenguas funcionales diferentes que, muchas veces funcionan obstaculizando la comunicación.

Tabla1

\begin{tabular}{|l|l|}
\hline Habla dialogal automática & $\begin{array}{l}\text { Masas aperceptivas } \\
\text { Alumnos/profesores }\end{array}$ \\
\hline $\begin{array}{l}\text { Se entiende según los supuestos } \\
\text { (apercepciones) }\end{array}$ & No coincidencia naturalmente \\
\hline $\begin{array}{l}\text { Las nociones técnicas vs el } \\
\text { sentido común }\end{array}$ & Espacio a construir desde el profesor \\
\hline
\end{tabular}

Además, el concepto de habla dialogal automática puede relacionarse con el espacio verbal/mental de planificación de las consignas de trabajo (Riestra, 2008).

Si al elaborar las consignas de trabajo para los alumnos utilizamos conceptos técnicos, sabemos que es necesario reformular esas consignas y explicar los conceptos que, en muchos casos obturan la comunicación. La distancia entre el contexto de recepción de nuestros alumnos y nuestro contexto profesional es grande; puede observarse tanto a nivel del léxico, como a nivel comunicativo; dicho de otro modo: los estereotipos técnico-conceptuales que naturalizamos funcionan como malentendidos o equívocos, sin que podamos darnos cuenta en el contexto de la clase.

Como un aporte teórico-práctico, en el marco de la perspectiva interaccionista socio-discursiva, nos planteamos analizar las consignas dentro del diálogo de la clase como contexto comunicativo más amplio.

Cuando los docentes nos disponemos a elaborar las consignas, enfocamos, por lo general, el objeto de enseñanza y descuidamos lo dialogal automático que implica nuestros estereotipos (en particular, los de la jerga técnica de los docentes de lengua) sin prestar atención a las masas aperceptivas de los alumnos a los que nos dirigimos.

Entendemos que la consigna (Tabla 2) se formula pensando en los destinatarios alumnos y sus particularidades concretas como edad, año, contexto singular del curso, contenidos enseñados y algunas capacidades ya operacionalizadas con las que suponemos que ellos cuentan. Ese paso de la planificación es el de prestar atención al interlocutor y su reacción frente a nuestro enunciado. 
Revista de la Escuela de Ciencias de la Educación, año 14, nRo. 13, vol. 1, enero a Junio de 2018. Páginas 167-179. ISSN 1851-6297 (DESDE DICIEMBRE DE 2006 A DICIEMBRE DE 2017). ISSN 2362-3349 (EN LiNEA). LA INTERACCIÓN EN LA CLASE DE LENGUA Y LITERATURA: ANÁLISIS DEL DIÁLOGO. DoRA RIESTRA.

Tabla 2

\section{Consignas}

El docente dice para que los alumnos hagan

Es un espacio verbal y mental

El docente se sitúa en la mente del interlocutor-alumno para formular verbalmente la tarea que deberán realizar los alumnos

Este es un primer espacio de análisis que implica varias tareas didáctico-pedagógicas como:

a) Pensar (durante la planificación de las consignas) en las posibles reformulaciones, o, dicho de otro modo, en las posibles traducciones de nuestra jerga técnico-lingüística para pasar desde el espacio dialogal mediatizado (desde la masa aperceptiva del otro/alumno/s) al espacio monologal escrito $u$ oral, que es artificial -según Jakubinskij- y complejo.

b) El objeto de estudio escolar en su pasaje por el habla dialogal encuentra una aprobación o reprobación de parte de los alumnos. El análisis del diálogo nos permitiría observarlo.

c) Nos preguntamos acerca de cómo prever o, por lo menos, atender a la masa aperceptiva de nuestros alumnos concretos, qué creen que les pedimos que hagan y sobre todo, "para qué".

Se trata, en definitiva de articular el plano de la praxis con el plano epistémico, o dicho de otro modo, el hacer verbal (la praxis del lenguaje o "lenguajear", según Maturana, 2003) con el conocimiento técnico que pretendemos que internalicen y se apropien del mismo. El tomar conciencia de las diferentes lenguas funcionales que ponemos en juego en la clase es el objetivo principal.

Para este artículo presentamos el análisis de una clase de primer año de secundario (de un total de seis clases), de una secuencia didáctica organizada en el género (textual) literario "cuento de terror". Como mencionamos antes, se trató de la aplicación de una propuesta didáctica diseñada por integrantes del equipo de investigación.

A partir del estudio del diálogo de Jakubinskij, el aporte de Voloshinov respecto del habla dialogal consiste en considerar el carácter extraverbal de su conformación, la producción social del diálogo y las formas concretas que llamó "géneros de la palabra". Para él "un enunciado concreto (no es una abstracción lingüística) nació, vivió y murió en el proceso de interacción social de los participantes" (Voloshinov, 1926/ Ivanova, 2010).

Por lo tanto, puede sostenerse que en el concepto de habla dialogal ruso está el germen del concepto de "género de la palabra", delimitado y desarrollado en Marxismo y filosofía del lenguaje (1929) por Valentin Voloshinov, quien fuera alumno de Jakubinskij entre 1923 y 1926, en estudios de posgrado, según Ivanova (2010; 2015). 
Revista de la Escuela de Ciencias de la Educación, año 14, nRo. 13, vol. 1, enero a junio de 2018. Páginas 167-179. ISSN 1851-6297 (DESDE DICIEMBRE DE 2006 A DICIEMBRE DE 2017). ISSN 2362-3349 (EN LiNEA). LA INTERACCIÓN EN LA CLASE DE LENGUA Y LITERATURA: ANÁLISIS DEL DIÁLOGO. DORA RIESTRA.

El concepto de género textual que asumimos teóricamente, con las consecuencias prácticas que se derivan del mismo (Riestra, 2010, 2014), se basa en los desarrollos teóricos de Voloshinov (2009). Según Ivanova:

Al desarrollar sus reflexiones sobre la interacción social y su papel en la producción de un enunciado, sobre la influencia del auditorio en la forma del enunciado, Voloshinov llega a la idea de los géneros verbales que unas veces denomina "géneros de la vida cotidiana" (zitejskie zanry) y otras "géneros verbales de la vida" (ziznnenye recevye zanry). En todos los casos la noción base es "género de la vida cotidiana (ustojcivajabytovaja situacija)" (Ivanova, 2010, p.58).

El concepto de análisis del diálogo fue formulado 30 años antes de las teorías llamadas pragmáticas en el contexto anglófono, es decir, el enfoque pragmático del enunciado se produjo entre los rusos sin que Europa central lo reconociera o lo entendiera.

En occidente el malentendido epistemológico se produjo al traducir como "género discursivo" lo que en ruso no existe conceptualmente (el concepto de discurso), por lo que es traducible por lingüistas eslavistas (Sériot e Ivanova, 2015) como "genre de la parole", que en español traducimos como "género de la palabra o del habla". Esto significa que en las traducciones al francés de los años 60 se colocó el concepto en el nivel del análisis lingüístico únicamente, cuando, particularmente, el mismo Voloshinov (1929/2009) lo situó en tres niveles simultáneos: el nivel comunicativo, el nivel del contenido y el nivel lingüístico, como tres dimensiones de la actividad verbal que se producen e inscriben, necesariamente, en un contexto concreto de comunicación.

Hecha la aclaración respecto del concepto de género, enfocamos el diálogo de la clase introductoria del género que la docente denomina "cuento de terror", un género literario bastante utilizado didácticamente, en función de las preferencias de los alumnos, tanto de primario, como de secundario. No nos detendremos en el sentido de la elección de este género, aunque pudo notarse (según las transcripciones etnográficas de la clase observada y analizada) que los estudiantes de primer año de la escuela pública de Bariloche, donde se desarrolló la secuencia didáctica, tenían conocimiento previo del género literario, lo que indicaría que en el nivel primario también habían leído cuentos de terror.

La complejidad de la enseñanza de la lengua y la literatura puede resumirse en la Tabla 3, que presenta el objeto de enseñanza (textos en géneros con sus propiedades y organización gramatical específica) y los tipos discursivos como las posibilidades verbales disponibles en los alumnos, con su mayor o menor dominio lingüístico (observable en su lengua funcional) y en el medio, articulando lo praxiológico y lo epistémico, situamos el diálogo como espacio de análisis semiológico y, además, como análisis del trabajo docente. 
Revista de la Escuela de Ciencias de la Educación, año 14, nRo. 13, vol. 1, enero a Junio de 2018. Páginas 167-179. ISSN 1851-6297 (DESDE DICIEMBRE DE 2006 A DICIEMBRE DE 2017). ISSN 2362-3349 (EN LiNEA). LA INTERACCIÓN EN LA CLASE DE LENGUA Y LITERATURA: ANÁLISIS DEL DIÁLOGO. DoRA RIESTRA.

Tabla 3

\begin{tabular}{|l|l|l|}
\hline Géneros textuales & Diálogo profesor-alumno & Tipos discursivos \\
\hline $\begin{array}{l}\text { Organización textual y } \\
\text { propiedades genéricas }\end{array}$ & $\begin{array}{l}\text { Masas aperceptivas } \\
\text { diferentes }\end{array}$ & $\begin{array}{l}\text { Operaciones psico- } \\
\text { verbales }\end{array}$ \\
\hline $\begin{array}{l}\text { Comprensión y } \\
\text { producción de textos }\end{array}$ & $\begin{array}{l}\text { Signos lingüísticos } \\
\text { (unidad semiológica de } \\
\text { Saussure, 2004) }\end{array}$ & $\begin{array}{l}\text { Lengua funcional o } \\
\text { Dialecto social de } \\
\text { los alumnos }\end{array}$ \\
\hline
\end{tabular}

\section{El análisis del diálogo}

Para mostrar el análisis del diálogo de esta clase de primer año, en primer lugar, en la Tabla 4, se parte de las masas aperceptivas coincidentes entre la profesora y los alumnos, resaltando momentos de las interacciones de la clase que ponen de relieve cómo los alumnos están pendientes de la lectura de un texto hecho por una alumna que concita la atención de todos. Dice la docente: "Sigo, esperen, hay un 3, ¿qué significa eso? Un signo" (se trata de un dibujo- emoticón- en medio del texto): La alumna aclara: "Es una carita". La docente continúa leyendo y concluye: "Está así, no estoy agregando nada" (aplausos). El aplauso general posterior de los alumnos es un gesto de aprobación y una muestra de atención ante esa lectura, por lo que en ese momento las apercepciones son coincidentes y la docente puede proponer una tarea de escritura, que será bien recibida, ante la valoración positiva del texto escrito por una alumna. En consecuencia, todos los alumnos reciben con buena disposición la tarea cuando la docente dicta la consigna de escritura: "Ya que están inspirados con la escritura vamos a hacer una actividad de escritura cuando terminemos. Vamos a sacar una hoja en blanco para hacer la actividad de escritura en el día de hoy".

Lo mismo sucede con las réplicas de la tercera columna, se trata de preguntas y respuestas de significados con intensa e interesada participación de los alumnos sobre el sentido del texto de Quiroga conocido por ellos. 
Revista de la Escuela de Ciencias de la Educación, año 14, nRo. 13, vol. 1, enero a junio de 2018. Páginas 167-179. ISSN 1851-6297 (DESDE DICIEMBRE DE 2006 A DICIEMBRE DE 2017). ISSN 2362-3349 (EN LiNEA). LA INTERACCIÓN EN LA CLASE DE LENGUA Y LITERATURA: ANÁLISIS DEL DIÁLOGO. DORA RIESTRA.

Tabla 4

\begin{tabular}{|c|c|c|c|}
\hline $\begin{array}{l}\text { Apercepciones } \\
\text { coincidentes }\end{array}$ & $\begin{array}{l}\text { Bien, voy con la } \\
\text { lectura (saca celu- } \\
\text { lar a una alumna). } \\
\text { Empiezo: Mi nombre } \\
\text { es Dalma Rayen } \\
\text { Figueroa [...] (Hacen } \\
\text { comentarios, docen- } \\
\text { te continúa leyendo). } \\
\text { Sigo, esperen, hay } \\
\text { un } 3 \text {, ¿qué significa } \\
\text { eso? Un signo. } \\
9.40 \\
\text { Aa: Una carita. } \\
\text { D: Qué ignorancia, } \\
\text { por Dios, sigo (lee) } \\
\text { Resaltado acá. Bue- } \\
\text { no, Dalma, estabas } \\
\text { inspirada. RÉPLICA } \\
\text { (la alumna aclara). } \\
\text { D: Está así, no estoy } \\
\text { agregando nada } \\
\text { (aplauden). Toda- } \\
\text { vía sigue. Cantaba } \\
\text { pero... ¿de quién es? } \\
\text { Aa: No me acuerdo, } \\
\text { son parte de una } \\
\text { canción. } \\
\text { (aplausos) }\end{array}$ & $\begin{array}{l}\text { D:Ya que están } \\
\text { inspirados con } \\
\text { la escritura } \\
\text { vamos a hacer } \\
\text { una actividad } \\
\text { de escritura } \\
\text { cuando termi- } \\
\text { nemos. Vamos } \\
\text { a sacar una } \\
\text { hoja en blanco } \\
\text { para hacer la } \\
\text { actividad de } \\
\text { escritura en el } \\
\text { día de hoy". }\end{array}$ & $\begin{array}{l}\text { Aa: Cuentos } \\
\text { de la selva. } \\
\text { D: ¿Qué es } \\
\text { insomnio? } \\
\text { Aa: No po- } \\
\text { dés dormir. } \\
\text { D: Otra pala- } \\
\text { bra... } \\
\text { Ao: Hipoté- } \\
\text { tica } \\
\text { D: ¿Qué es? } \\
\text { Aa: Algo que } \\
\text { vos creés. } \\
\text { Te imaginás } \\
\text { que algo va } \\
\text { a pasar. }\end{array}$ \\
\hline $\begin{array}{l}\text { Apercepciones } \\
\text { no coincidentes }\end{array}$ & $\begin{array}{l}\text { Natalia, ¿está?, } \\
\text { ¿querés leer?, nos } \\
\text { quedamos sin re- } \\
\text { creo, dale Natalia } \\
\text { (intenta leer).Bien, } \\
\text { Natalia, no se en- } \\
\text { tiende porque no se } \\
\text { escucha. ¡Timbre! } \\
\text { Mañana seguimos. }\end{array}$ & $\begin{array}{l}\text { Brenda, mante- } \\
\text { nete sentada... } \\
(10.30) \\
\text { Brenda corta- } \\
\text { la...(10.40) }\end{array}$ & $\begin{array}{l}\text { Escribítu } \\
\text { idea antes } \\
\text { de que se } \\
\text { te vaya, si } \\
\text { no, el pobre } \\
\text { lector va a } \\
\text { quedar pen- } \\
\text { sando. } \\
\text { Ao: ¿Qué } \\
\text { lector? } \\
\text { D: El que lee } \\
\text { el cuento. }\end{array}$ \\
\hline
\end{tabular}


Revista de la Escuela de Ciencias de la Educación, año 14, nRo. 13, vol. 1, enero a Junio de 2018. Páginas 167-179. ISSN 1851-6297 (DESDE DICIEMBRE DE 2006 A DICIEMBRE DE 2017). ISSN 2362-3349 (EN LiNEA). LA INTERACCIÓN EN LA CLASE DE LENGUA Y LITERATURA: ANÁLISIS DEL DIÁLOGO. DoRA RIESTRA.

Así como en el momento de las lecturas las masas aperceptivas coinciden, en otros momentos de la clase los alumnos no atienden, hablan en lugar de escuchar, a pesar de que una alumna intenta leer su producción, no es posible, la docente superpone su voz, pero no es escuchada; pueden notarse las diferencias en los supuestos de la profesora y un alumno en la tercera columna: la docente se refiere al pobre lector (en abstracto) y un alumno pregunta qué lector (en concreto), ya que no imagina otro lector de su texto que la misma docente, por lo que ella debe aclarar que se trata del que lee el texto, función teórica, aunque la noción técnica de la profesora no fue registrada por el alumno en su lengua funcional, es decir, desde el sentido común; ambos parten de percepciones conceptuales diferentes frente al lector del texto del que hablan. Se trata de un implícito que la docente no aclara porque su suposición generalizante del concepto de lector no es tomada por el alumno en ese momento de la tarea (aquí puede notarse con precisión la diferencia de lengua funcional o sociolecto entre docente y alumno).

Por otra parte, los estereotipos o fases hechas de la clase, llamando la atención, son indicadores interesantes para la autorreflexión docente en este tipo de análisis, como se ve en la Tabla 5, porque muestra las veces que recurre automáticamente a enunciados como llamados de atención para que se sienten los alumnos o para que escriban, con el consecuente desgaste que implica para la docente.

Por otra parte, las réplicas estereotipadas de alumnos señalan la ausencia de compromiso frente a la tarea de la clase y la necesidad de ser escuchados por los demás alumnos.

Tabla 5

\begin{tabular}{|l|l|l|l|l|}
\hline $\begin{array}{l}\text { Estereotipos } \\
\text { docentes }\end{array}$ & $\begin{array}{l}\text { Nos quedamos } \\
\text { leyendo en el } \\
\text { recreo, aviso... } \\
\text { Nos quedamos } \\
\text { sin recreo... }\end{array}$ & $\begin{array}{l}\text { Belén, en } \\
\text { tu lugar }\end{array}$ & $\begin{array}{l}\text { S e b a s - } \\
\text { tián, a tu } \\
\text { lugar }\end{array}$ & $\begin{array}{l}\text { Jacqueline, } \\
\text { Milagros, } \\
\text { sentate }\end{array}$ \\
\hline $\begin{array}{l}\text { Estereotipos } \\
\text { alumnos }\end{array}$ & $\begin{array}{l}\text { Vamos a jugar al } \\
\text { futbol }\end{array}$ & $\begin{array}{l}\text { ¿Qué } \\
\text { mierda } \\
\text { digo? }\end{array}$ & & \\
\hline
\end{tabular}

Cabe señalar que en el análisis de las consignas realizado no es posible observar estos aspectos que, desde el análisis del diálogo, muestran las apercepciones y sirven para entender estrictamente la interacción entre docente y alumnos desde la comunicación, un aspecto complementario del análisis didáctico de los objetos de enseñanza que, en nuestro caso, fue el cuento de 
Revista de la Escuela de Ciencias de la Educación, año 14, nRo. 13, vol. 1, enero a junio de 2018. Páginas 167-179. ISSN 1851-6297 (DESDE DICIEMBRE DE 2006 A DICIEMBRE DE 2017). ISSN 2362-3349 (EN LiNEA). LA INTERACCIÓN EN LA CLASE DE LENGUA Y LITERATURA: ANÁLISIS DEL DIÁLOGO. DORA RIESTRA.

terror y las formas gramaticales correspondientes.

En las seis clases la docente enfocó como objeto a ser enseñado el género literario como formato social conocido por los alumnos y partió de los tipos discursivos que, como operaciones psico-verbales en constante movilización, los alumnos tienen adquiridos (saben contar porque han escuchado y leído cuentos en ese género, como señalamos antes). Por lo tanto, la propuesta didáctica partió de una escritura espontánea en primer lugar y otras propuestas de escritura posteriores con consignas más específicas sobre verbos y adjetivos. Los alumnos lograron escribir tres textos a lo largo de seis clases, lo que es objeto de otro tipo de análisis didáctico: el efecto de la enseñanza a partir de los textos producidos por los alumnos.

En este artículo pretendimos mostrar cómo la cuestión didáctica de articulación de las lenguas funcionales o dialectos sociales de los alumnos con su determinismo apercetivo, que incluye prejuicios, desinterés, aburrimiento, etc, puede articularse o no con la lengua funcional de nosotros los docentes, con nuestros supuestos y simplificaciones.

En la progresión de las clases pudo observarse cómo la docente explicó las categorías gramaticales de adjetivo y verbo y los alumnos lograron identificar semiológicamente la función gramatical.

Observamos, a modo de síntesis, que este proceso de enseñanza-aprendizaje desigual en el desarrollo de los alumnos no puede analizarse ni generalizarse enfocando la transposición didáctica.

Por lo tanto, desde la investigación, nos interesa incorporar y articular los dos tipos de análisis: por una parte, el análisis didáctico de las consignas con los objetos de enseñanza y, por otra, el análisis del trabajo docente desde esta perspectiva de análisis del diálogo, que hemos comenzado a instrumentar metodológicamente.

Si bien, como sostiene Jakubinskij (2015) las palabras pasan al segundo plano en el diálogo, consideramos que la revisión del diálogo de la clase puede colaborar en la autorreflexión del docente para lograr el efecto buscado con su planificación.

La finalidad del análisis del diálogo es la de ofrecer un dispositivo de utilidad profesional. Es en esta dirección que estamos comenzando a elaborar el instrumento para probarlo en situaciones concretas, a partir de las transcripciones de observaciones etnográficas.

Una finalidad más general de esta propuesta metodológica es la de aportar a la profesionalidad de la formación en la didáctica específica, que se contrapone a la supuesta naturalidad del espontaneísmo. Para esto, el principio que nos orienta es paradojal: necesitamos escuchar atentamente la lengua funcional de los alumnos para introducirlos en nuestra lengua funcional como profesores de lengua y literatura.

La disociación entre los contenidos de lengua y los de literatura podrá su- 
Revista de la Escuela de Ciencias de la Educación, año 14, nRo. 13, vol. 1, enero a Junio de 2018. Páginas 167-179. ISSN 1851-6297 (DESDE DICIEMBRE DE 2006 A DICIEMBRE DE 2017). ISSN 2362-3349 (EN LiNEA). LA INTERACCIÓN EN LA CLASE DE LENGUA Y LITERATURA: ANÁLISIS DEL DIÁLOGO. DoRA RIESTRA.

perarse a partir de coincidencias entre las masas aperceptivas de profesores y alumnos.

El estudio del diálogo pudo mostrar que los contenidos artísticos son inherentes a la posibilidad de comunicación, que las reflexiones estilístico-gramaticales carecen de sentido sin una percepción compartida de supuestos, prejuicios, valoraciones, etc.

De este modo, el aporte metodológico centrado en el análisis del diálogo, esbozado aquí en la perspectiva del análisis del trabajo docente en el aula, tiene la finalidad de que nuestros conocimientos profesionales incidan en los procesos de desarrollo de los alumnos.

\section{Referencias bibliográfícas}

- Bertau, M.C. (2008). Pour une notion de forme linguistique comme forme vécue. Une approche avec Jakubinskij, Volosinov et Vygotski in Langage et pensée: Union sovietique années 1920-1930. Lausanne: UNIL.

- Bronckart, J. P. (2002). La explicación en Psicología ante el desafío del significado. Estudios de Psicología, 23 (3) pp.387-416.

- Bronckart, J. P. (2007). Desarrollo del lenguaje y didáctica de las lenguas. Buenos Aires: Miño y Dávila.

- Bronckart, J.P. (2004). Actividad verbal, textos y discursos. Por un interaccionismo socio-discursivo. Madrid: Infancia y Apendizaje.

- Bronckart, J.P. (1997). Activité langagière, textes et discours. Pour un interactionisme Coseriu, E. (1992). Competencia lingüística. Madrid: Gredos. socio-discursif. Paris: Delachaux et Niestlé

- Ivanova, I. (2010)El diálogo en la lingüística soviética de los años 1920-1930. En: Riestra, D. Saussure, Voloshinov y Bajtin revisitados. Estudios históricos y epistemológicos. Buenos Aires: Miño y Dávila

- Ivanova, I. (2012). Lev Jakubinskij, une linguistique de la parole Lausanne: Lambert Lucas

- Jakubinskij, L. (2015). Sobre a fala dialogal. Ed. Sériot e Ivanova. Sao Paulo: Parábola.

- James, W. (1994). Principios de psicología. México: Fondo de Cultura Económica.

- Paas, F.; Renkl, A. y Sweller, J. (2004). Cognitive Load Theory: Instructional Implications of the Interaction between Information Structures and Cognitive Architecture. Instructional Science 32: 1-8, 2004. Kluwer Academic Publishers. Printed in the Netherlands

- Riestra, D. (2008). Las consignas de enseñanza de la lengua. Buenos Aires: Miño \& Dávila

- Riestra, D. (2010). Saussure, Voloshinov y Bajtin revisitados. Estudios históricos y epistemológicos. Buenos Aires: Miño \& Dávila.

- Riestra, D., Goicoechea, M.V. y Tapia, S. M. (2014). Los géneros textuales en secuencias didácticas de Lengua y Literatura. Buenos Aires: Noveduc.

- Sacks, H.; Shegloff, E.; Jefferson, G. (1974). A simplest systematic for the organization of turn-talking for conversation. Language, Vol 50, n 4, part 1. (dec. 1974) pp 696-735.

- Voloshinov, V. (2009). Marxismo y filosofia del lenguaje. Buenos Aires:Godot 